\title{
Formation of resonant bonding during growth of ultrathin GeTe films
}

\author{
Ruining Wang ${ }^{1}$, Wei Zhang ${ }^{2}$, Jamo Momand ${ }^{3}$, Ider Ronneberger ${ }^{4,5}$, Jos E Boschker ${ }^{1}$, Riccardo Mazzarello ${ }^{4,5}$, \\ Bart J Kooi ${ }^{3}$, Henning Riechert ${ }^{1}$, Matthias Wuttig ${ }^{5,6}$ and Raffaella Calarco ${ }^{1}$
}

A highly unconventional growth scenario is reported upon deposition of GeTe films on the hydrogen passivated Si(111) surface. Initially, an amorphous film forms for growth parameters that should yield a crystalline material. The entire amorphous film then crystallizes once a critical thickness of four GeTe bilayers is reached, subsequently following the $\mathrm{GeTe}(111) \| \mathrm{Si}(111)$ : GeTe [-110] || Si[-110] epitaxial relationship rigorously. Hence, in striking contrast to conventional lattice-matched epitaxial systems, a drastic improvement in atomic order is observed above a critical film thickness. Raman spectra show a remarkable change of vibrational modes above the critical thickness that is attributed to a change in the nature of the bonds: While ordinary covalent bonding is found in ultrathin films, resonant bonding can prevail only once a critical thickness is reached. This scenario is further supported by density functional theory calculations showing that ultrathin films do not utilize resonant bonding in contrast to the bulk phase. These findings are important not only for ultrathin films of phase-change materials such as GeTe and $\mathrm{GeSbTe}$, which are employed in phase-change memories, but also for thermoelectrics and topological insulators such as $\mathrm{Bi}_{2} \mathrm{Te}_{3}$ and $\mathrm{Sb}_{2} \mathrm{Te}_{3}$, where resonant bonding might also have a significant role.

NPG Asia Materials (2017) 9, e396; doi:10.1038/am.2017.95; published online 30 June 2017

\section{INTRODUCTION}

Innovations in materials synthesis often enable breakthroughs in realizing novel technologies. This is exemplified by heterostructure band engineering with epitaxial superlattices in optoelectronics and information technology. ${ }^{1}$ Hence, concepts to grow epitaxial films of excellent quality have a crucial role in semiconductor physics. Recently, epitaxial growth has also been reported for a class of resonantly bonded chalcogenide compounds, including $\mathrm{GeTe}, \mathrm{Sb}_{2} \mathrm{Te}_{3}$ and GeSbTe alloys (GST). ${ }^{2-4}$ Resonant bonding in chalcogenides is a unique bonding mechanism, which differs significantly from metallic or ordinary covalent bonding. The atoms in crystalline compounds such as GeTe or elemental Sb have to a first approximation six nearest neighbors but only three $p$ electrons per atom to form saturated bonds, sometimes called two center-two electron bonds. Therefore, there are too many nearest neighbors for the electrons to form ordinary covalent bonds to each neighbor. Instead, the system exhibits a different bonding configuration: it employs three center-two electron bonds, also denoted as resonant bonds. In this situation, neighboring atoms are held together just by a single electron, not an electron pair. Unlike in metals, however, these electrons are still rather localized between two atoms, leading to a non-vanishing band gap. Resonant bonding can also be described as a superposition of electronic configurations featuring two center-two electron bonds, somewhat resembling the electronic configuration in, for example, benzene. ${ }^{5}$ Resonant bonding in chalcogenides is accompanied by characteristic features, which include large values of the Born effective charge $\left(Z^{*}\right)$ and the optical dielectric constant $\left(\varepsilon_{\infty}\right){ }^{6}$ Indeed, the transition from the amorphous to the crystalline state in materials such as $\mathrm{GeTe}$ or $\mathrm{Ge}_{2} \mathrm{Sb}_{2} \mathrm{Te}_{5}$ is accompanied by a significant increase of $\varepsilon_{\infty}$ and $Z^{*} \cdot{ }^{6-8}$ This observation is in line with a transition from ordinary covalent bonding in the amorphous state, where the atoms have on average three nearest neighbors, ${ }^{9}$ that are held by saturated bonds, to resonant bonding in the crystalline state.

Chalcogenides such as $\mathrm{GeTe}, \mathrm{Sb}_{2} \mathrm{Te}_{3}$ or $\mathrm{Ge}_{2} \mathrm{Sb}_{2} \mathrm{Te}_{5}$ are important as phase-change materials, thermoelectrics, topological insulators or ferroelectrics. ${ }^{10-13}$ With novel applications for these materials within reach, epitaxial growth provides the best platform to understand how their different properties are manifested in an ultrathin film. Some interesting examples are the investigation of Anderson localization in ultrathin $\left(\mathrm{Bi}_{1-x} \mathrm{Sb}_{x}\right)_{2} \mathrm{Te}_{3}$ topological insulators ${ }^{14}$ or the surprising increase of the Curie temperature in ultrathin SnTe films compared with the bulk, enabling stable macroscopic in-plane polarization at room temperature in a two-unit-cell-thick film. ${ }^{15} \mathrm{SnTe}$ is closely related to $\mathrm{GeTe}$, but different phenomena were observed during the downscaling of the thickness in the latter, with surface preparation having a predominant role. ${ }^{16} \mathrm{~A}$ number of studies have briefly

${ }^{1}$ Paul-Drude-Institut für Festkörperelektronik, Berlin, Germany; ${ }^{2}$ Center for Advancing Materials Performance from the Nanoscale, State Key Laboratory for Mechanical Behavior of Materials, Xi'an Jiaotong University, Xi'an, China; ${ }^{3}$ Zernike Institute for Advanced Materials, University of Groningen, Groningen, The Netherlands; ${ }^{4}$ Institute for Theoretical Solid State Physics, RWTH Aachen University, Aachen, Germany; ${ }^{5}$ JARA-FIT and JARA-HPC, RWTH Aachen University, Aachen, Germany and ${ }^{6}$. Physikalisches Institut, RWTH Aachen University, Aachen, Germany

Correspondence: Dr R Calarco, Paul-Drude-Institut für Festkörperelektronik, Hausvogteiplatz 5-7, Berlin 10117, Germany.

E-mail: calarco@pdi-berlin.de

Received 15 November 2016; revised 13 February 2017; accepted 24 March 2017 
a

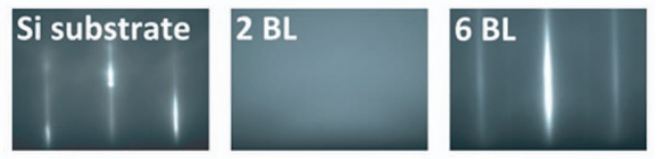

b
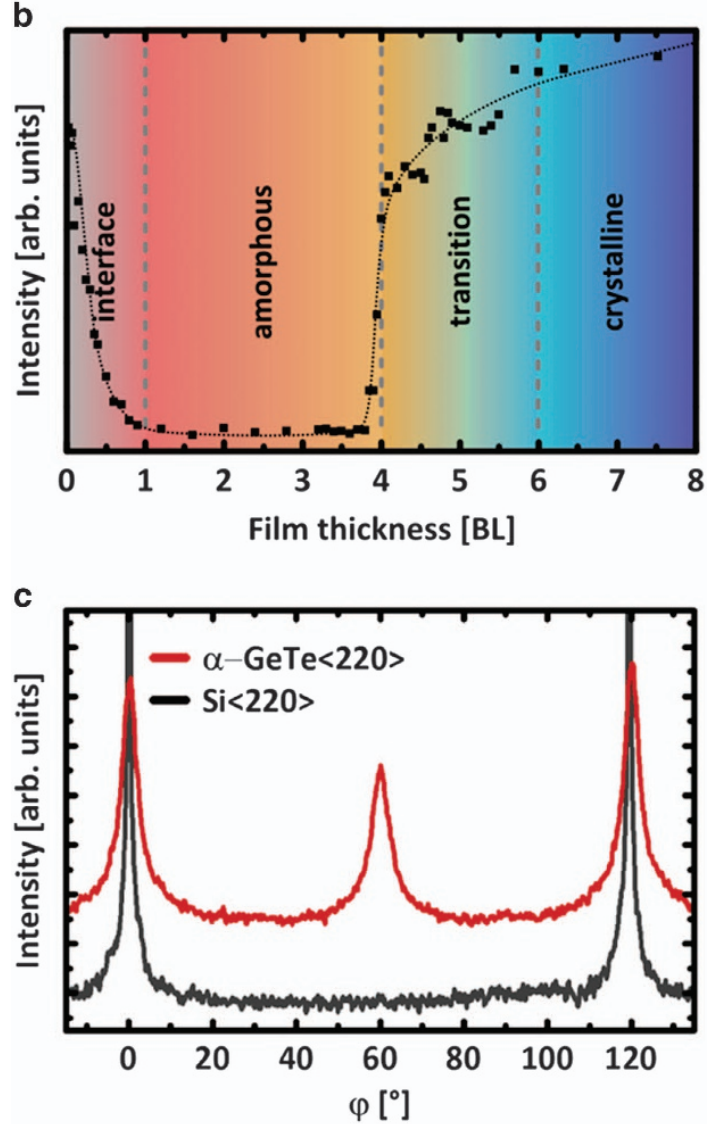

d

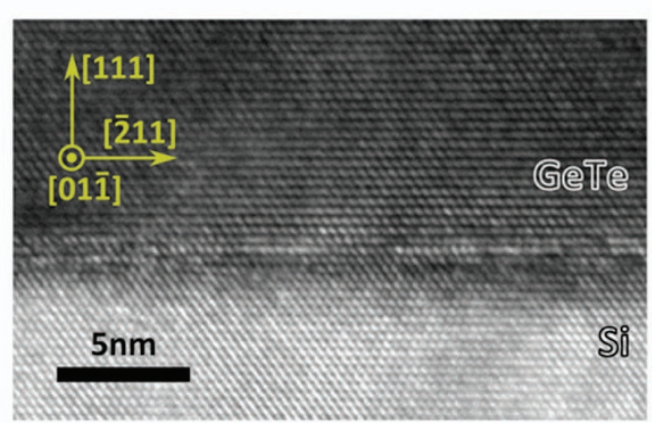

Figure 1 (a) RHEED images along $<-110>$ azimuth during growth of GeTe on $\mathrm{Si}(111)-(1 \times 1)-\mathrm{H}$ surface. These images are taken before deposition and at 2 and $6 \mathrm{BL}$ nominal thickness. The GeTe film is first amorphous and crystallizes during growth. (b) Integrated RHEED intensity during growth around the $\mathrm{GeTe}(111)-(1 \times 1)$ reconstruction streaks, showing that crystallization occurs after formation of 4 GeTe BL. (c) XRD $\varphi$-scan around $\mathrm{Si}(111)$ axis, aligned on the $\mathrm{GeTe}(220)$ reflections, Si(220) reflections are shown as the reference. Once the film crystallizes, a strong in-plane epitaxial relationship is found with the substrate. (d) Cross-sectional TEM image showing the $\{-110\}$ lattice planes in a $20 \mathrm{~nm}$ thick GeTe thin-film grown on $\mathrm{Si}(111)-(1 \times 1)-\mathrm{H}$. The film is fully crystalline with a sharp interface, showing no misfit dislocations. mentioned the growth of chalcogenides starting with the formation of non-crystalline layers, ${ }^{2,4,17,18}$ whereas the growth of a crystalline phase is observed from the very beginning in other cases. ${ }^{19,20}$ These differences are puzzling and motivate further studies. In the following, the growth of ultrathin GeTe films deposited by molecular beam epitaxy on the $\mathrm{Si}(111)-(1 \times 1)-\mathrm{H}$ surface is investigated. Subsequently, we investigate the decisive factors which govern the atomic arrangement by density function theory (DFT) calculations and by comparison with previous results on the $\mathrm{Si}(111)-(\sqrt{ } 3 \times \sqrt{ } 3) \mathrm{R} 30^{\circ}-\mathrm{Sb}$ surface.

\section{MATERIALS AND METHODS}

\section{Experimental details}

All the substrates are cleaned using the method described in Wang et al. ${ }^{19}$ where the preparation of the $\mathrm{Si}(111)-(\sqrt{ } 3 \times \sqrt{ } 3) \mathrm{R} 30-\mathrm{Sb}$ surface is also described. As for the $\mathrm{Si}(111)-(1 \times 1)-\mathrm{H}$ surface, it is obtained by dipping the $\mathrm{Si}$ (111) substrates in a $10 \%$ buffered hydrofluoric acid solution for $10 \mathrm{~min}$, stripping the oxide layer and passivating the surface with hydrogen. ${ }^{21}$ The typical $(1 \times 1)$ surface reconstruction is observed by RHEED (shown in Figure 1a) and holds even after the first $20 \mathrm{~s}$ of GeTe deposition, showing the robustness of the passivation. The growth itself is performed at a substrate temperature of $260^{\circ} \mathrm{C}$, using $\mathrm{Ge}$ and Te dual-filament effusion cells with base and tip temperature of $T_{\text {base }}(\mathrm{Ge})=1120^{\circ} \mathrm{C}$ and $T_{\text {tip }}(\mathrm{Ge})=1140^{\circ} \mathrm{C}$ for the $\mathrm{Ge}$ cell, $T_{\text {base }}(\mathrm{Te})=335^{\circ} \mathrm{C}$ and $T_{\text {tip }}(\mathrm{Te})=470{ }^{\circ} \mathrm{C}$ for the Te cell. The cell fluxes are calibrated beforehand by performing X-ray reflectivity measurements on amorphous $\mathrm{Ge}$ and $\mathrm{Te}$ films grown at room temperature. The cell temperatures used presently correspond to deposition rates at room temperature of $0.17 \mathrm{~nm} \mathrm{~min}{ }^{-1}$ for Ge and $0.4 \mathrm{~nm} \mathrm{~min}{ }^{-1}$ for Te, resulting in a Ge/Te flux ratio of $\sim 2 / 5$. At the end of the growth, the sample is cooled down to room temperature, and prior to its removal from the molecular beam epitaxy, the sample is capped with $10-15 \mathrm{~nm}$ of $\mathrm{Si}_{3} \mathrm{~N}_{4}$ by RF sputtering in the molecular beam epitaxy load-lock chamber in order to prevent oxidation. The diffractometer used for X-ray diffraction (XRD) characterization of the samples is a Panalytical X'Pert PRO MRD system with Ge (220) hybrid monochromator (Panalytical, Kassel, Germany), employing a CuKo $-1 \quad(\lambda=1.5406 \AA)$ X-ray radiation. Raman measurements were performed on a HORIBA LabRAM HR Evolution system (Horiba, Villeneuve-d'Ascq, France) in $z(y, x y)-z$ geometry with a $633 \mathrm{~nm}$ laser. Transmission electron microscopic specimens were prepared by mechanical grinding and ion-polishing with a Gatan PIPS II (Gatan Inc., Pleasanton, CA, USA). Cross-sectional high-resolution transmission electron microscopic imaging was performed using a JEOL 2010F (JEOL Ltd., Tokyo, Japan).

\section{Simulation details}

DFT simulations are performed using the Quantum Espresso package. ${ }^{22}$ Ultrasoft $^{23}$ and norm-conserving ${ }^{24}$ pseudopotentials are employed for GeTe and $\mathrm{GaSb}$, respectively. We use GGA-PBE ${ }^{25}$ functionals but include van der Waals corrections by adopting the Grimme's D2 method. ${ }^{26}$ The wave-function cutoff is set to $60 \mathrm{Ry}$ for GaSb models and $50 \mathrm{Ry}$ for GeTe models, for which the charge density cutoff is set to $500 \mathrm{Ry}$. The Brillouin zone is sampled by using a $20 \times 20 \times 20(20 \times 20 \times 1) k$-point mesh $^{27}$ for the relaxation calculations of bulk (thin-layer) models. For the self-consistent calculations prior to phonon calculations, a finer $40 \times 40 \times 40($ resp. $40 \times 40 \times 1) k$-point mesh is used. We include dipole corrections ${ }^{28}$ for the thin-layer simulations to eliminate the spurious field in the vacuum region due to the finite polarization of the models. Phonon calculations are performed using the density functional perturbation theory method. ${ }^{29}$ The phonon frequencies at the zone center are computed by taking into account the non-analytical correction arising from the finite polarization. The dielectric constant provided by our slab calculations is not well defined because it depends on the volume of the supercell, including the vacuum slab. To extract an effective two-dimensional dielectric constant, we employ the formula used by Gomes et al. ${ }^{30} \varepsilon_{\infty}=1+\left(\varepsilon_{\infty}{ }^{\text {DFT }}-1\right) \times L_{c} /\left(n \times L_{\text {bilayer }}\right)$, where $\varepsilon_{\infty}{ }^{D F T}$ is the dielectric constant yielded by the DFT calculation, $n$ is the number of bilayers (BLs), $L_{\mathrm{c}}$ is the length of the supercell along the direction perpendicular to the slab and $L_{\mathrm{bilayer}}$ is the $\mathrm{BL}$ spacing in bulk GeTe or GaSb, 

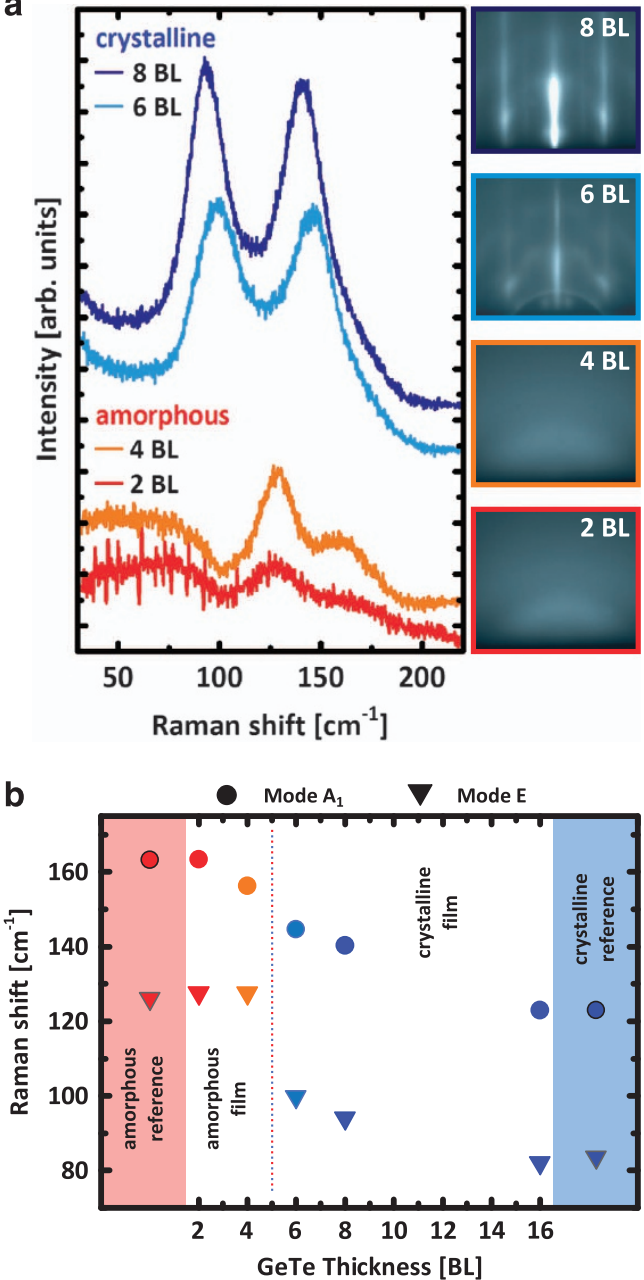

Figure 2 (a) Raman spectra acquired on GeTe samples of 2, 4, 6 and 8 BL nominal thickness grown on the $\mathrm{Si}(111)-(1 \times 1)-\mathrm{H}$ surface. RHEED images acquired at the end of each growth are also shown. Significant differences in intensity and frequency of the Raman modes are observed for the two thinner samples, which are amorphous, as compared with the two thicker crystalline samples. (b) The position of the observed Raman modes are reported as a function of thickness. The values obtained on a $30 \mathrm{~nm}$ thick crystalline GeTe film and reference values for amorphous $\mathrm{GeTe}^{31}$ are shown as well. Although the spectra of the 6 and $8 \mathrm{BL}$ sample closely resemble the crystalline (bulk-like) reference sample, the spectrum for the $4 \mathrm{BL}$ sample are quite similar to the amorphous reference sample. The pronounced change of the frequency of the Raman modes between 4 and $6 \mathrm{BL}$ is indicative for a change in bonding mechanism upon crystallization.

equal to 3.50 and $3.51 \AA$, respectively. All data calculated with this model are gathered in Supplementary Table SI-1, including an additional case for a GeTe slab on top of a $\mathrm{Sb}(111)$ template.

\section{RESULTS AND DISCUSSION}

Figure 1a shows RHEED images recorded during the growth of GeTe on the Si(111) $-(1 \times 1)-\mathrm{H}$ surface. The first image characterizes the starting surface. Within the deposition of the first GeTe BL (1 $\mathrm{BL}=0.35 \mathrm{~nm}$ ), the substrate streaks fade out, leaving only diffusely scattered intensity, as shown on the second image. Because there is a mismatch of $8.5 \%$ between $\mathrm{GeTe}$ and $\mathrm{Si}^{19}{ }^{19}$ reflections from both materials are easily distinguished by RHEED, and it is clear that no crystalline GeTe is formed up to this point, the surface is covered by an amorphous material instead. After the deposition of four BLs, new streaks corresponding to the $\mathrm{GeTe}(111)-(1 \times 1)$ surface appear and remain until the end of the growth at a thickness of $\sim 30 \mathrm{~nm}$. Hence, the GeTe film first grows with an amorphous structure and then crystallizes as a thickness of four BLs is reached, as further illustrated by Figure $1 \mathrm{~b}$ where the integrated intensity near the position expected for the GeTe streaks is plotted during growth. As the new streaks appear, their spacing already corresponds to that of $\alpha-\mathrm{GeTe}$, which indicates that the film is relaxed.

An XRD $\varphi$-scan around the $\mathrm{Si}(111)$ axis is performed, aligned on the $\mathrm{GeTe}(220)$ reflections, as shown in Figure 1c. Single peaks spaced by $60^{\circ}$ are measured and aligned with the silicon substrate reflections, which means that the GeTe film follows an in-plane epitaxial relationship $\mathrm{GeTe}(-110) \| \mathrm{Si}(-110)$. Similarly to GeTe grown on $\mathrm{Si}$ $(111)-(\sqrt{ } 3 \times \sqrt{ } 3) \mathrm{R} 30^{\circ}-\mathrm{Sb},{ }^{19}$ the single peaks demonstrate that the otherwise favorable in-plane rotational twists at $\pm 2^{\circ}$ and $\pm 7^{\circ}$ related to domain matching epitaxy are suppressed..$^{2}$ There is still dispersion in the texturing, as evidenced by the full width at half maximum of the reflections, but the distribution of twisted domains becomes unimodal and aligned along the substrate azimuths. The intervals of $60^{\circ}$ between the threefold symmetric $\{220\}$ reflections show that twinning is also present.

In the out-of plane direction, the symmetric $2 \theta-\omega$ XRD scan (not shown here) reveals that the same high-quality out-of-plane texture is also obtained as for GeTe films deposited on $\operatorname{Si}(111)-(\sqrt{ } 3 \times \sqrt{ } 3) \mathrm{R}^{\circ} 0^{\circ}-\mathrm{Sb}$ surface, ${ }^{3,19}$ and $\mathrm{Si}(111)-(7 \times 7){ }^{2}$ To further illustrate the excellent quality of the film, cross-sectional transmission electron microscopic analysis in Figure $1 \mathrm{~d}$ shows that the film is indeed crystalline, with a sharp interface and no signs of strain fields, hence no presence of misfit dislocations. The observation of such crystalline quality and well-defined out-of plane epitaxial relationship is surprising, considering that the film originated from the crystallization of an amorphous phase.

Thus there is a remarkable difference between the initial stage of the growth on the $\mathrm{Si}(111)-(1 \times 1)-\mathrm{H}$ surface, compared with the growth on $\mathrm{Si}(111)-(\sqrt{ } 3 \times \sqrt{ } 3) \mathrm{R} 30^{\circ}-\mathrm{Sb}$ that yielded a crystalline phase for all film thicknesses. ${ }^{3,19}$ To further investigate this divergence, GeTe films with nominal thickness of two and four BLs were prepared, where the RHEED pattern still showed no crystalline streaks at the end of the growth. Two thicker samples are then grown until the RHEED streaks have appeared, with nominal thicknesses of six and eight BLs. In Figure 2a, the Raman spectrum for each sample is presented along with the corresponding RHEED image acquired at the end of the growth. In all cases, the RHEED pattern remains unchanged after the deposition is stopped, demonstrating that both the amorphous and crystalline ultrathin films are stable.

For the two thicker samples, two modes of similar intensity can be identified at 94 and $140.3 \mathrm{~cm}^{-1}$ for the eight BLs sample and 99.6 and $144.6 \mathrm{~cm}^{-1}$ for the six BLs. They correspond to the $(\mathrm{E})$ and $\left(\mathrm{A}_{1}\right)$ modes of $\alpha-G e T e$ at 83 and $123 \mathrm{~cm}^{-1}$ but strengthened due to the impact of the predominating interfaces. ${ }^{32}$ All films being capped with $\mathrm{Si}_{3} \mathrm{~N}_{4}$ in order to prevent oxidation, both the interface with the substrate and capping layer contribute to the shift of the Raman modes.

The two spectra for the thinner films look qualitatively different. Modes are observed at 128.6 and $156.2 \mathrm{~cm}^{-1}$ for the four BL sample, while they are found at 127.4 and $163.3 \mathrm{~cm}^{-1}$ for the two BL sample, and their intensity ratio is about $2 / 3$. These modes match very well with literature values for amorphous GeTe at 127 and $162 \mathrm{~cm}^{-1} .31$ The presence of a broad Bose peak near the $50 \mathrm{~cm}^{-1}$ range also indicates that an amorphous layer is formed during the initial stage of growth. ${ }^{33}$ A summary of the observed Raman frequencies 


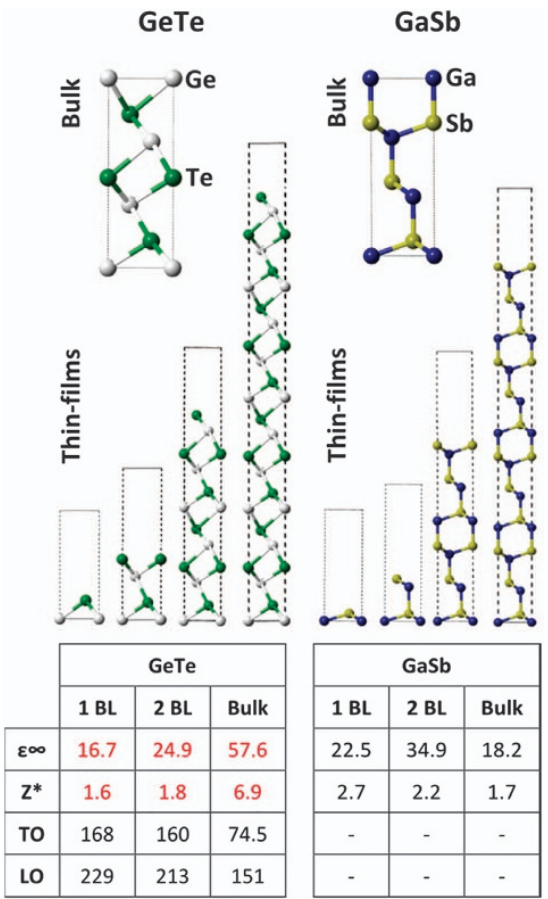

Figure 3 The bulk and thin-layer models of GeTe and GaSb. All structures are fully relaxed with respect to cell parameters and atomic positions with inclusion of van der Waals corrections. ${ }^{24}$ All slab models contain a $20 \AA$ vacuum slab. The calculated values for $\varepsilon_{\infty}, Z^{*}$ and the two predicted Raman modes in each cases are reported below their corresponding schematic. For $\mathrm{GeTe}$, ultrathin films show very low values of $\varepsilon_{\infty}$ and $Z^{*}$, incompatible with resonant bonding, while the bulk sample possesses this characteristic fingerprint (large values of $\varepsilon_{\infty}$ and $Z^{*}$ ).

is plotted in Figure 2b as a function of the film thickness. While the higher frequency mode seems to shift linearly toward the (E) mode of crystalline GeTe, the lower frequency mode displays a clear change in its position between four and six BLs. Such a pronounced difference between the Raman spectra for the four and six BL sample is very striking and requires an explanation, as crystallization in most solids is only accompanied by rather modest changes of the position and width of vibrational modes. Interesting enough, previous investigations have already shown a significant difference of the Raman spectrum for much thicker amorphous and crystalline samples of the same compound. This change of vibrational modes is a characteristic fingerprint of phase-change materials. The pronounced difference of the Raman spectra has been attributed to a change of bonding mechanism from covalent bonding in the amorphous phase to resonant bonding in the crystalline phase. ${ }^{6,7,34,35}$ The Raman mode positions for amorphous and crystalline GeTe are shown for comparison in Figure 2b. The Raman modes measured in the four BL (amorphous) film match with those in the amorphous reference, while the spectrum for the six BL thick (crystalline) film is very similar to the spectrum of the resonantly bonded crystalline sample. This provides strong evidence that the thinner films exhibit ordinary covalent bonding, while the thicker films above four BLs show resonant bonding. Once the film is crystalline, neither the Bose peak nor modes of the amorphous GeTe are observed, ruling out the possibility for parts of the film to remain amorphous or to have crystalline material growing on top of a subsisting amorphous layer. The latter scenario would also be incompatible with the epitaxial nature of the thicker films. The scenario observed here hence also differs significantly from the case of delayed crystallization of $\mathrm{Gd}_{2} \mathrm{O}_{3}$ on $\mathrm{Si}(111),{ }^{36}$ where no such change in bonding is observed.

It is interesting that both Raman modes that are measured in the ultrathin amorphous GeTe film can be attributed to the vibration of atoms in defective octahedral sites. ${ }^{37}$ The modes at frequencies $>185 \mathrm{~cm}^{-1}$ corresponding to homopolar Ge-Ge bonds in tetrahedral structures $^{38}$ are not observed. Considering that Raty et al. ${ }^{39}$ have demonstrated that the relaxation of amorphous GeTe leads to the reduction of these less stable bonds and that the amorphous GeTe in the present ultrathin film is slowly deposited at a high temperature of $260^{\circ} \mathrm{C}$, homopolar $\mathrm{Ge}-\mathrm{Ge}$ bonds and associated tetrahedral structures are apparently strongly suppressed.

The next step is to understand why resonant bonding only prevails above a critical thickness on specific surface reconstructions. The rearrangement of the entire film for constant growth temperature indicates that the transition is governed by energetic constraints. Resonant bonding can best be described by a stabilization of a compound through electron delocalization. This is similar to the idea that metallic bonding is favored due to a decrease in kinetic energy upon electron delocalization. Hence, in the ultrathin geometry, electron delocalization in the direction of the film normal can be strongly impaired, if the interfaces create an electronic barrier.

In the comparison between $\mathrm{Si}(111)-(1 \times 1)-\mathrm{H}$ and $\mathrm{Si}(111)-$ $(\sqrt{3} \times \sqrt{3})-S b,{ }^{3,19}$ where GeTe is able to form a crystalline thin-film directly, the presence of $\mathrm{Sb}$ stands out as the main difference. The exact mechanism by which resonant bonding is enabled in this case is still subject to further investigation. $\mathrm{Sb}$ does have a natural tendency for resonant bonding, both in its pure form and also when intermixed with GeTe into GST, ${ }^{10}$ and the Sb-rich environment could be promoting the formation of resonant bonds. But the $\mathrm{Sb}$ passivating the $\mathrm{Si}(111)$ surface only form one single atomic layer that is covalently bonded via $s p^{3}$ hybridization. Therefore, the Sb in this specific case does not simply form a 'resonantly bonded template'. But in contrast with the H-passivation, the Sb-passivation leaves a highly directional lone pair pointing upwards, ${ }^{40}$ whereas all electrons are mostly concentrated below the $\mathrm{H}$ atoms in the former. This difference in the nature of the passivation could influence the stability of the amorphous and crystalline phases of GeTe deposited above.

Finally, the pronounced change of bonding in ultrathin GeTe films is investigated by DFT calculations. Freestanding models were built along the [111] direction of GeTe with a vacuum slab of $20 \AA$, as shown in Figure 3. A detailed description of the model is given in the Supplementary Information section. Following the calculation approach for the dielectric function of two-dimensional systems by Gomes et al., ${ }^{30}$ tremendous differences in $\varepsilon_{\infty}$ and $Z^{*}$ are found between a freestanding film of one or two BLs of GeTe and a bulk phase. The characteristic fingerprint of resonant bonding, that is, high values of $\varepsilon_{\infty}$ and $Z^{\star}$ are not observed for ultrathin films. This clearly indicates that the ultrathin films do not utilize resonance bonding, in contrast to the bulk phase, which shows very large values of $\varepsilon_{\infty}$. These thin films show a more pronounced band gap in DFT calculations, similar to the amorphous state of bulk phase-change materials; this is further evidence for ordinary covalent bonding. ${ }^{6}$ Even if the atoms of the one or two BL films are forced within the model into the same positions as the bulk, no resonant bonding is formed. This last aspect is also reflected in the Raman data of the amorphous phase at growth onset showing predominantly defective octahedral structures: Even though the atoms are locally arranged into the 
right geometrical configuration for resonant bonding, the material does not manage to delocalize its $p$ electrons to form the resonantly bonded network.

This behavior of freestanding GeTe films is strikingly different from the scenario observed for freestanding films of GaSb, a covalently bonded system ( $s p^{3}$ bonded), where both the atomic positions and the dielectric function do not differ significantly from the corresponding bulk phase. The dielectric properties of one BL GeS, GeSe, SnS and SnSe have been shown to be very close to their bulk states as well. ${ }^{30}$ Although these systems are also are bonded with three $p$ electrons per atom in average, no resonant bonding is present due to the misalignment of their $p$ orbitals. ${ }^{41,42}$ This is further evidence for our claim that only resonantly bonded solids show unconventional properties as a thin film, while this effect is not observed for $s p^{3}$-bonded materials (for example, GaSb) or for $p$-bonded solids with angular disorder (for example, GeSe), which do not utilize resonant bonding. Finally, the calculated phonon properties can be compared with the data measured in the ultrathin GeTe films. Qualitatively, both the transverse and the longitudinal optical modes decrease significantly as the film thickness increases, in line with the trend observed in the experimental data. A quantitative comparison should not be drawn, as the presence of the substrate and capping material are neglected in the model.

It is interesting that confinement in only one direction is sufficient to cause a drop in $\varepsilon_{\infty}$ and $Z^{\star}$, disrupting the resonant bonds, when the thin-film geometry imposes in principle no constrains in-plane. Also in the case of $\mathrm{Ge}_{2} \mathrm{Sb}_{2} \mathrm{Te}_{5}(100)$ grown on $\mathrm{GaSb}(100)$, where $p$ orbitals are expected in the film plane, the same phenomenon of an amorphous transition at growth onset was also reported. ${ }^{4}$ This shows that the three resonant $p$ orbitals are interdependent and that electron delocalization has to be possible in all three directions for resonant bonding to exist.

\section{CONCLUSION}

In conclusion, two distinct growth mechanisms for GeTe are identified depending on the surface treatment of silicon and hence the electronic interaction between the growing film and the substrate. Although it is difficult to isolate and quantify this electronic interaction from an experimental point of view, this study highlights the critical importance of the interfaces for the promotion or suppression of resonant bonding inside an ultrathin layer. Because resonant bonding is the signature and the very origin of the many properties in these chalcogenide compounds, those findings are not only relevant for scaling purposes in phase-change properties but also for the synthesis of thermoelectric superlattices, topological insulators and the novel class of ferroelectric Rashba semiconductors. ${ }^{43}$

\section{CONFLICT OF INTEREST}

The authors declare no conflict of interest.

\section{ACKNOWLEDGEMENTS}

We thank S Behnke and C Stemmler for technical support at the molecular beam epitaxy system. JMJ Lopes is acknowledged for careful reading of the manuscript. This work was partially supported by EU within the FP7 project PASTRY (GA 317746) and by the Leibniz Gemeinschaft within the Leibniz Competition on a project titled: Epitaxial phase-change superlattices designed for investigation of non-thermal switching. We gratefully acknowledge the computational resources provided by the HPCC Platform, Xi'an Jiaotong University and the support by JARA-HPC from RWTH Aachen University under project JARA0142. Funding through SFB 917 (Nanoswitches) to MW and RM and through an ERC Advanced Grant 340698 ('Disorder control') to MW is also acknowledged. WZ gratefully thanks the support of the Youth
Thousand Talents Program of China and the Young Talent Support Plan of Xi'an Jiaotong University.

Author contributions: RW performed the growth and characterization of GeTe. Analysis of data was mostly carried out by RW with the support from JEB. MW strongly contributed to the model of resonant bonding. WZ and IR performed DFT simulations and analyzed the data together with RM and MW. HRTEM characterization was performed by JM and BJK. The paper was written by RW, RC and MW, with the help and through contributions from all coauthors. All authors have given approval to the final version of the manuscript. The project was initiated and conceptualized by RC.

1 Alfero, Z. I. \& Kroemer, H. Nobel Lectures in Physics 1996-2000 (World Scientific Publishing Company, Singapore, 2003).

2 Giussani, A., Perumal, K., Hanke, M., Rodenbach, P., Riechert, H. \& Calarco, R. On the epitaxy of germanium telluride thin films on silicon substrates. Phys. Status Solidi 249, 1939-1944 (2012).

3 Boschker, J. E., Momand, J., Bragaglia, V., Wang, R., Perumal, K., Giussani, A. Kooi, B. J., Riechert, H. \& Calarco, R. Surface reconstruction-induced coincidence lattice formation between two-dimensionally bonded materials and a threedimensionally bonded substrate. Nano Lett. 14, 3534-3538 (2014).

4 Braun, W., Shayduk, R., Flissikowski, T., Ramsteiner, M., Grahn, H. T., Riechert, H., Fons, P. \& Kolobov, A. V. Epitaxy of Ge-Sb-Te phase-change memory alloys. Appl. Phys. Lett. 94, 41902 (2009).

5 Pauling, L. The nature of chemical bond. Application of results obtained from a theory of parmagnetic susceptibility to the structure of molecules. J. Am. Chem. Soc. 53, 1367-1400 (1931).

6 Shportko, K., Kremers, S., Woda, M., Lencer, D., Robertson, J. \& Wuttig, M. Resonant bonding in crystalline phase-change materials. Nat. Mater. 7, 653-658 (2008).

7 Lucovsky, G. \& White, R. M. R. Effects of resonance bonding on the properties of crystalline and amorphous semiconductors. Phys. Rev. B 8, 660 (1973).

8 Chen, C., Jost, P., Volker, H., Kaminski, M., Wirtssohn, M., Engelmann, U., Krüger, K., Schlich, F., Schlockermann, C., Lobo, R. P. S. M. \& Wuttig, M. Dielectric properties of amorphous phase-change materials. Phys. Rev. B 95, 094111 (2017).

9 Kolobov, A. V., Fons, P., Frenkel, A. I., Ankudinov, A. L., Tominaga, J. \& Uruga, T. Understanding the phase-change mechanism of rewritable optical media. Nat. Mater. 3, 703-708 (2004).

10 Lencer, D., Salinga, M., Grabowski, B., Hickel, T., Neugebauer, J. \& Wuttig, M. A map for phase-change materials. Nat. Mater. 7, 972-977 (2008).

11 Kim, Y., DiVenere, A., Wong, G. K. L., Ketterson, J. B., Cho, S. \& Meyer, J. R. Structural and thermoelectric transport properties of $\mathrm{Sb}[$ sub 2]Te[sub 3] thin films grown by molecular beam epitaxy. J. Appl. Phys. 91, 715 (2002).

12 Pauly, C., Liebmann, M., Giussani, A., Kellner, J., Just, S., Sanchez-Barriga, J., Rienks, E., Rader, O., Calarco, R., Bihlmayer, G. \& Morgenstern, M. Evidence for topological band inversion of the phase change material Ge2Sb2Te5. Appl. Phys. Lett. 103, 243109 (2013).

13 Kolobov, A. V., Kim, D. J., Giussani, A., Fons, P., Tominaga, J., Calarco, R. \& Gruverman, A. Ferroelectric switching in epitaxial GeTe films. APL Mater. 2, 66101 (2014).

14 Liao, J., Ou, Y., Feng, X., Yang, S., Lin, C., Yang, W., Wu, K., He, K., Ma, X., Xue, Q.-K. \& $\mathrm{Li}, \mathrm{Y}$. Observation of Anderson localization in ultrathin films of three-dimensional topological insulators. Phys. Rev. Lett. 114, 216601 (2015).

15 Chang, K., Junwei, L., Haicheng, L., Wang, N., Zhao, K., Zhang, A., Jin, F., Zhong, Y., Hu, X., Duan, W., Zhang, Q., Fu, L., Xue, Q.-K., Chen, X. \& Ji, S.-H. Discovery of robust in-plane ferroelectricity in atomic-thick SnTe. Science 353, 274-278 (2016).

16 Wang, R., Campi, D., Bernasconi, M., Momand, J., Kooi, B. J., Verheijen, M. A., Wuttig, M. \& Calarco, R. Ordered Peierls distortion prevented at growth onset of GeTe ultra-thin films. Sci. Rep. 6, 32895 (2016).

17 McCarthy, D. N., Yaginuma, S., Gui, H. \& Nagao, T. Thickness dependent phase transition of $\mathrm{Bi}$ films quench condensed on semiconducting surfaces. CrystEngComm 13, 4604 (2011)

18 Hashimoto, M., Niizeki, T. \& Kambe, K. Effect of substrate temperature on crystallization of amorphous antimony film. Jpn J. Appl. Phys. 19, 21-23 (1980).

19 Wang, R., Boschker, J. E., Bruyer, E., Sante, D., Di, Picozzi, S., Perumal, K., Giussani, A., Riechert, H. \& Calarco, R. Toward truly single crystalline GeTe films: the relevance of the substrate surface. J. Phys. Chem. C 118, 29724-29730 (2014).

20 Katmis, F., Calarco, R., Perumal, K., Rodenbach, P., Giussani, A., Hanke, M., Proessdorf, A., Trampert, A., Grosse, F., Shayduk, R., Campion, R., Braun, W. \& Riechert, $H$. Insight into the growth and control of single-crystal layers of Ge-Sb-Te phase-change material. Cryst. Growth Des. 11, 4606-4610 (2011).

21 Vinh, L. T., Eddrief, M., Sébenne, C. A., Dumas, P., Taleb-Ibrahimi, A., Gunther, R., Chabal, Y. J. \& Derrien, J. Low temperature formation of Si(111)7 $\times 7$ surfaces from chemically prepared H/Si(111)-(1×1) surfaces. Appl. Phys. Lett. 64, 3308 (1994).

22 Giannozzi, P., Baroni, S., Bonini, N., Calandra, M., Car, R., Cavazzoni, C., Ceresoli, D., Chiarotti, G. L., Cococcioni, M., Dabo, I., Dal Corso, A., de Gironcoli, S., Fabris, S., Fratesi, G., Gebauer, R., Gerstmann, U., Gougoussis, C., Kokalj, A., Lazzeri, M., Martin-Samos, L., Marzari, N., Mauri, F., Mazzarello, R., Paolini, S., Pasquarello, A., Paulatto, L., Sbraccia, C., Scandolo, S., Sclauzero, G., Seitsonen, A. P., Smogunov, A., 
Umari, P. \& Wentzcovitch, R. M. QUANTUM ESPRESSO: a modular and open-source software project for quantum simulations of materials. J. Phys. Condens. Matter 21, 395502 (2009).

23 Vanderbilt, D. Soft self-consistent pseudopotentials in a generalized eigenvalue formalism. Phys. Rev. B 41, 7892-7895 (1990).

24 Hartwigsen, C., Goedecker, S. \& Hutter, J. Relativistic separable dual-space Gaussian pseudopotentials from H to Rn. Phys. Rev. B 58, 3641 (1998).

25 Perdew, J. P., Burke, K. \& Ernzerhof, M. Generalized gradient approximation made simple. Phys. Rev. Lett. 77, 3865-3868 (1996).

26 Grimme, S. Semiempirical GGA-type density functional constructed with a long-range dispersion correction. J. Comput. Chem. 27, 1787-1799 (2006).

27 Monkhorst, H. J. \& Pack, J. D. Special points for Brillouin-zone integrations. Phys. Rev. B 13, 5188-5192 (1976).

28 Bengtsson, L. Dipole correction for surface supercell calculations. Phys. Rev. B 59, 12301-12304 (1999).

29 Baroni, S., De Gironcoli, S., Dal Corso, A. \& Giannozzi, P. Phonons and related crystal properties from density-functional perturbation theory. Rev. Mod. Phys. 73, 515-562 (2001).

30 Gomes, L. C. \& Carvalho, A. Phosphorene analogues: isoelectronic two-dimensional group-IV monochalcogenides with orthorhombic structure. Phys. Rev. B 92, 85406 (2015)

31 Andrikopoulos, K. S., Yannopoulos, S. N., Voyiatzis, G. A., Kolobov, A. V., Ribes, M. \& Tominaga, J. Raman scattering study of the a-GeTe structure and possible mechanism for the amorphous to crystal transition. J. Phys. Condens. Matter 18, 965-979 (2006).

32 Yu, N. N., Tong, H., Zhou, J., Elbashir, A. A. \& Miao, X. S. Local order of Ge atoms in amorphous GeTe nanoscale ultrathin films. Appl. Phys. Lett. 103, 61910 (2013).

33 Andrikopoulos, K. S., Yannopoulos, S. N., Kolobov, A. V, Fons, P. \& Tominaga, J. Raman scattering study of GeTe and Ge2Sb2Te5 phase-change materials. J. Phys. Chem. Solids 68, 1074-1078 (2007).

34 Sosso, G C., Caravati, S., Gatti, C., Assoni, S. \& Bernasconi, M. Vibrational properties of hexagonal $\mathrm{Ge}(2) \mathrm{Sb}(2) \mathrm{Te}(5)$ from first principles. J. Phys. Condens. Matter 21, 245401 (2009).

35 Matsunaga, T., Yamada, N., Kojima, R., Shamoto, S., Sato, M., Tanida, H., Uruga, T, Kohara, S., Takata, M., Zalden, P., Bruns, G., Sergueev, I., Wille, H. C., Hermann, R. P. \& Wuttig, M. Phase-change materials: vibrational softening upon crystallization and its impact on thermal properties. Adv. Funct. Mater. 21, 2232-2239 (2011).

36 Hanke, M., Kaganer, V. M., Bierwagen, O., Niehle, M. \& Trampert, A. Delayed crystallization of ultrathin Gd2O3 layers on $\mathrm{Si}(111)$ observed by in situ X-ray diffraction. Nanoscale Res. Lett. 7, 203 (2012).

37 Mazzarello, R., Caravati, S., Angioletti-Uberti, S., Bernasconi, M. \& Parrinello, M. Signature of tetrahedral Ge in the Raman spectrum of amorphous phase-change materials. Phys. Rev. Lett. 104, 85503 (2010).

38 Deringer, V. L., Zhang, W., Lumeij, M., Maintz, S., Wuttig, M., Mazzarello, R. \& Dronskowski, R. Bonding nature of local structural motifs in amorphous GeTe. Angew. Chem. Int. Ed. Engl. 53, 10817-10820 (2014).

39 Raty, J. Y., Zhang, W., Luckas, J., Chen, C., Mazzarello, R., Bichara, C. \& Wuttig, M. Aging mechanisms in amorphous phase-change materials. Nat. Commun. 6, 7467 (2015).

40 Elswijk, H. B., Dijkkamp, D \& van Loenen, E. J. Geometric and electronic structure of $\mathrm{Sb}$ on $\mathrm{Si}(111)$ by scanning tunneling micrscopy. Phys. Rev. B 44, 3802 (1991).

41 Huang, B. \& Robertson, J. Bonding origin of optical contrast in phase-change memory materials. Phys. Rev. B 81, 81204 (2010).

42 Robertson, J. Mott lecture: how bonding concepts can help understand amorphous semiconductor behavior. Phys. Status Solidi 213, 1641-1652 (2016).

43 Di Sante, D., Barone, P., Bertacco, R. \& Picozzi, S. Electric control of the giant Rashba effect in bulk GeTe. Adv. Mater. 25, 509-513 (2013).

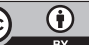

other third ird party material in this article are included in the article's Creative Commons license, unless indicated otherwise in the credit line; if the material is not included under the Creative Commons license, users will need to obtain permission from the license holder to reproduce the material. To view a copy of this license, visit http:// creativecommons.org/licenses/by/4.0/

(C) The Author(s) 2017

Supplementary Information accompanies the paper on the NPG Asia Materials website (http://www.nature.com/am) 